\title{
Coarse to Fine Level Set Segmentation of SAR Imagery Based on The Brovey Transform Fusion of Optical Imagery
}

\author{
P. Selvarani and V. Vaithyanathan \\ Sastra University, Thanjavur, India
}

\begin{abstract}
This study delivers a new approach for contour extraction in different terrains of the same location of both SAR images and optical images using a coarse to fine level set scheme. Segmentation in both SAR and optical imagery is a trivial challenge for many researchers. The main target is to segment buildings, lands and oil tanks perfectly via fusion with a level set method. First Brovey transform is applied in fusing both SAR images and optical images. Discrete wavelet transform is utilized for extracting an image from a background. Finally in order to segment an image a coarse to fine level set is implemented. Experiments have been carried out on quick bird and Terra SAR-x images to validate the proposed methodology.
\end{abstract}

Key words: Brovey fusion, coarse to fine level set, discrete wavelet transform, SAR image, optical image, contour extraction

\section{INTRODUCTION}

Earth observation from space technology has advanced enormously over the past two decades, particularly in the field of Microwave SAR (Synthetic Aperture Radar) and Optical Imagery. Both SAR and optical images are attaining at $1.0 \mathrm{~m}$ better resolution. The orbiting satellites have been improved a temporal resolution in constellation. Now due to the availability of these images, researchers should be able to obtain more gains in performance through the fusion of information contained in them. Data fusion helps to combine data attained by multiple sensors or the same sensor acquiring images at different times with an objective to obtain image of greater quality. In the remote sensing community they define it this: data fusion is a formal framework in which are explained means and tools for the coalition of data originating from different sources. It aims at obtaining information of greater quality; the exact definition of greater quality will depend upon the application of $\mathrm{N}$ sensors.

Synthetic aperture radar is defined as a form of radar which is used as a respective motion between an antenna and its victim region to provide a unique long-term coherent signal variation that is adventured to attain smaller spatial resolution than is potential with conventional beam. It emerged as a conquered contour of Side-Looking Airborne Radar (SLAR). SAR is usually executed by mounting on a pulpit such as an airliner or shuttle a unique transverse forming antenna from which a victim scene is repeatedly irradiated with pounds of radio waves at wavelengths anywhere from a metre down to millimetres. The many echo waveforms acquired consecutively at the peculiar antenna positions are systematically revealed and stored and then post-progressed together to design a component in an image of the focus region. The radar system yields a huge transmission capacity signal or otherwise usually in the structure of a chip. Remote sensing systems usually tune a transmission capacity of $10-30 \mathrm{MHz}$ but militant systems have a transmission capacity of $>500 \mathrm{MHz}$. Some sample original of SAR images are depicted in Fig. 1.
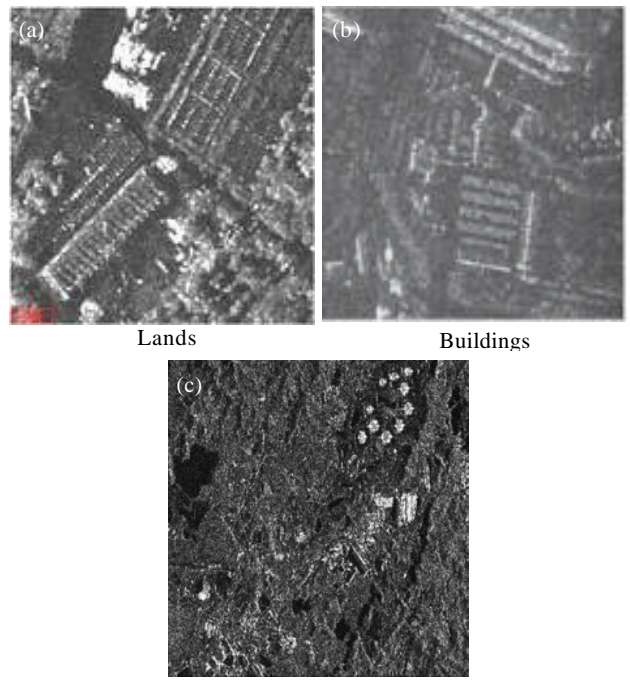

Oil tanks

Fig. 1a-c): The original SAR images

Corresponding Author: P. Selvarani, Sastra University, Thanjavur, India 


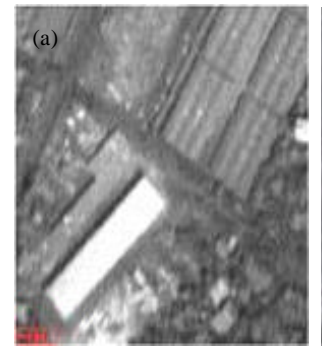

Lands

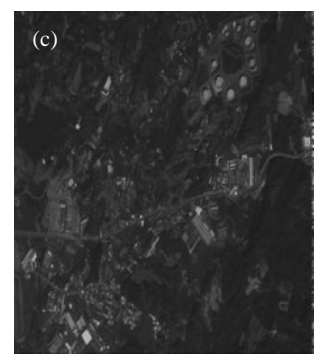

Oil tanks

Fig. 2a-c): The original optical images

Optical imaging is an imaging approach. Optics usually illustrates the activities of visual, ultraviolet and infrared light used in imagery because light is an electromagnetic wave, analogous circumstances which ensue in X-rays, microwaves, radio waves. Chemical or molecular imaging embraces inference from the deflection of light emanated from source to structure, texture an atomic and chemical properties of material. Optical imaging systems may be fragmented into diffusive and ballistic imaging systems. Some sample original optical images are depicted in Fig. 2.

There are three main contrasts of SAR over optical sensors. First both sensors use various frequencies or wavelengths. SAR requires a wavelength of $1 \mathrm{~cm}$ to $1 \mathrm{~m}$ to view through clouds and storms while optical sensors cannot be viewed because they use a wavelength beside that of visible light or 1 micron. Due to these two sensors an object on the earth's surface looks entirely different. This happens due to the scattering mechanism of electromagnetic waves are dependent on the wavelength. Secondly, to generate brightness, optical sensors decline upon the sun's illumination that is perceived by the sensor. In divergence an SAR sensor sustains its own radiance source in the structure of radio waves transmitted by an antenna. Thirdly, SAR sensors render angles to an object differently because SAR quantifies range rather than angle in the direction sheer to the line of flight (Lombardo et al., 2003; Yang et al., 2008; Tupin and Roux, 2003; Gamba et al., 2000; Christopher et al., 1997; Hellwich et al., 2000; Ayed et al.,
2005). Synthetic Aperture Radar (SAR) image segmentation is a key procedure in the automatic scrutinization and interpretation of data which can sustain the overall structural information on the image to divulge the essence of SAR images. Image segmentation establishes the foundation for Automatic Targets Recognition (ATR) and patronizes the wide application of SAR images.

SAR images and optical images are fused by Brovey fusion developed to perceivably increase the contrast in the low and high ends of an image histogram. Consequently, Brovey should not be tuned if perpetuating the original scene's radiometry is conspicuous. However, it is good for generating RGB (Red, Green and Blue) images with a higher degree of contrast in the low and high ends of an image's histogram.

Contour extraction is a challenging image segmentation problem in a computer vision. This problem is raised in shape based object recognition. The methods used in contour extraction problem are largely dependent on the features of the highly discriminative ability to distinguish an image from a background.

Coarse to fine level set is a suppositional technique which is used to segment an image perfectly. The main objective is to find complete $2 \mathrm{D}$ boundaries of the salient objects in both optical and SAR images. The advantage of the coarse to fine level set is to solve contour extraction problem.

The nominated method is to proficiently segment SAR and optical images where fusion is done via coarse to fine level set scheme. Brovey fusion is applied to increase the contrast of the image by merging both SAR and optical images. The fused image is passed to the discrete wavelet transform which is used for discriminating an image from a background. Finally, coarse to fine level set is implemented for perfect segmentation.

\section{MATERIALS AND METHODS}

Huaping et al. (2011) have proposed a method in order to solve contour extraction but they met with many difficulties. Law et al. (2008) have proposed a multi-stochastic level set method to solve contour extraction but it leads to the failure. Silveira and Heleno (2009) have proposed the probability distribution method in order to solve contour extraction problem but it also causes failure. Weisenseel et al. (1999) and Sarkar et al. (2002) have proposed a markov random field segmentation in solving contour extraction problem but it causes failure. 
In order to solve the contour extraction problem, coarse to fine level set can be used (Qizhi et al., 2011). The main contributions are illustrated as follows:

Brovey fusion: Brovey transform is a point type method. This method utilizes a ratio algorithm to fuse the images. It is attempted to generate RGB images. At a time, three bands can be synthesized from the multispectral image. The resultant image is then displayed with $R, G$ and $B$ bands to RGB. Brovey fusion is a simple fusion in order to achieve a better quality in an image. It is based on arithmetic multiplicative operations. It is similar to IHS (Intensity-Hue-Saturation) fusion. It is based on direct intensity modulation. Brovey works by splitting an image into color and intensity components. The intensity component can be replaced with a high resolution image. The purpose of Brovey fusion is used to merge both SAR image and optical image to upgrade the contrast of the image. It is used for generating RGB images with a higher degree of the image contrast. It is used to produce a visualizing image. The formulae can be calculated by using $\mathrm{R}, \mathrm{G}$ and $\mathrm{B}$ components.

$$
\begin{aligned}
& \text { Red }=R /(R+G+B) \times \text { pan } \\
& \text { Green }=G /(R+G+B) \times \text { pan } \\
& \text { Blue }=B /(R+G+B) \times \text { pan }
\end{aligned}
$$

The summation of the $R, G$ and $B$ values are equivalent to the intensity. The Eq. 1-3 can be rewritten as:

$$
\begin{gathered}
\text { Red }=\mathrm{R} / \mathrm{I} \times \text { pan } \\
\text { Green }=\mathrm{G} / \mathrm{I} \times \text { pan } \\
\text { Blue }=\mathrm{B} / \mathrm{I} \times \text { pan }
\end{gathered}
$$

By using Eq. 4-6 the images can be fused. The output of the fused images is shown in Fig. 3.

\section{Advantages:}

- It is based on arithmetic operation

- It is very fast

- It is even applicable to the large size images

- Spatial resolution of the image is efficient

Discrete wavelet transform: The purpose of the discrete wavelet transform is used to extract an image from a background. It has a shift invariant property. It produces sub bands of the same size as the input image. There are

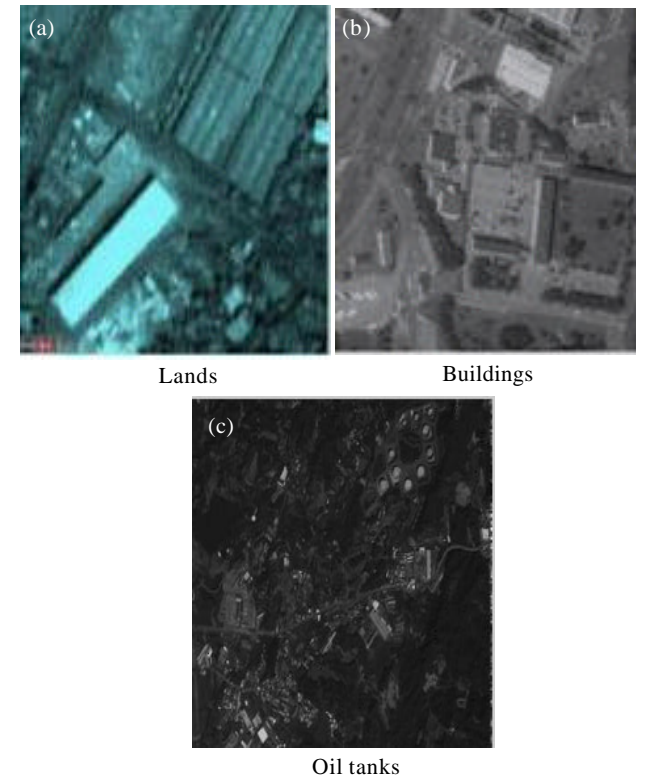

Fig. 3a-c): Brovey fusion output

3 kinds of the discrete wavelet transform. They are Haar, Daubechies and Hough wavelets. Here Haar wavelet is used in an image extraction.

Haar wavelet: Haar wavelet is a succession of rescaled square-shaped functions which consecutively design a wavelet category. Wavelet evaluation is analogous to Fourier analysis. It allows a victim function over an interlude to be referred in terms of an orthonormal function basis. The purpose of the Haar wavelet is used to determine the intensity difference of the image. Based on the intensity difference, Haar wavelet is used to extract an image from a background. Haar wavelet's calculation is done by the low pass filter and high pass filter. Low pass filter and high pass filter is denoted by $\mathrm{HO}, \mathrm{Hl}$, GO and G1. The values of the low pass filter are $0.5-0.5$. The values of the high pass filter are $0.5,0.5$. The output of the Haar wavelet is shown in Fig. 4.

\section{Advantages:}

- It is easy to implement

- It takes less time for decomposition

- Memory required in the process is efficient

Discrete wavelet weighing approach: The purpose of the homogeneity metric is used to calibrate the discrepancy of the images between inside and outside contours. Homogeneity metric's computation is done by the intensity contrast of the haar wavelet. The homogeneity metric is expressed by: 


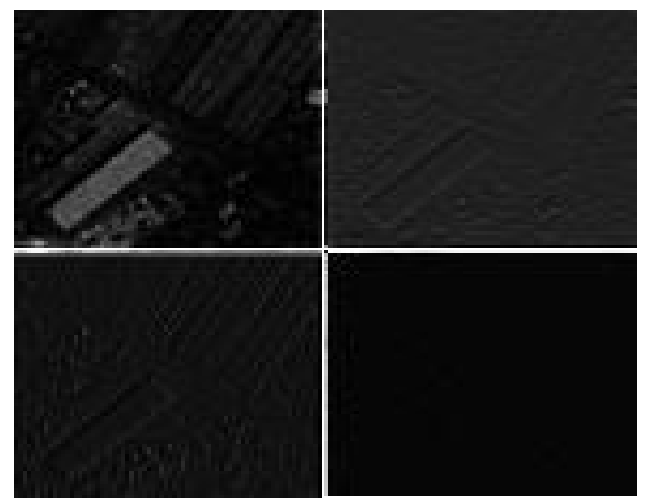

Fig. 4: Haar wavelet output

$$
\mathrm{E}\left(\mathrm{d}^{\mathrm{i}}, \Omega_{\mathrm{k}}\right)=\int\left(\mathrm{d}^{\mathrm{i}}(\mathrm{x}, \mathrm{y})-\mathrm{d}_{\mathrm{k}}^{\mathrm{i}}\right)^{2} \mathrm{dxdy}
$$

Discriminative ability $\left(\mathrm{d}^{\mathrm{i}}\right)$ is a component which is used to relate the homogeneous difference of the areas between inside and outside contours. Using a component an images discrepancy can be done perfectly. It is evaluated by the following expression:

$$
\eta\left(d^{i}, c\right)=\frac{E_{i, 0}+\varepsilon_{0}}{E_{i, 1}+E_{i, 2}+\varepsilon_{0}}
$$

where, $\mathrm{d}_{\mathrm{k}}^{\mathrm{i}}$ is the mean value of $\mathrm{d}^{\mathrm{i}}$ over region of $\mathrm{d}^{\mathrm{i}}$ over region $\Omega_{\mathrm{k}}$. Based on discriminative ability component, various weights are assigned to the feature components to separate an images from a background. This is known as weight distribution ratio. This ratio is computed by:

$$
\xi\left(d^{i}, c\right)=\frac{\eta\left(d^{i}, c\right)}{\sum_{j=1}^{4} \eta\left(d^{j}, c\right)}
$$

The corollary of the distinct wavelet weighing approach is novel energy function model. Novel energy function model is a structure which is used for solving contour extraction problem.

Coarse to fine level set segmentation: The purpose of this scheme is the reduction of the resolution level at a time. It is applied in a large evolution space which reduces to small space. It contains 3 modules:

- Coarse Scale Model

- Fine Scale Model

- Suppositional Model
Coarse Scale Model: Coarse Scale Model is used for depreciating the energy function. Energy function is formulated by plotting the weighted components such as haar wavelet, homogeneity metric and discriminative ability component and weight distribution ratio. It is expressed in terms of the equation:

$$
\begin{aligned}
(\mathrm{c})= & \mu \int|\nabla H \phi| d x d y+\sum \xi_{i, c} \int\left(d^{i}-\overline{d_{1}^{i}}\right)^{2} H_{\phi} d x d y+ \\
& \sum_{i=1}^{4} \xi_{i, c} \int\left(d^{i}-\overline{d_{2}^{i}}\right)^{2}\left(1-H_{\phi}\right) d x d y
\end{aligned}
$$

Fine Scale Model: Contour position constraint is introduced for miniaturizing the contour evolution space to a small region. It is measured by the space between boundaries of the images. It is obtained by the following equation:

$$
\mathrm{R}_{\alpha}(\mathrm{x}, \mathrm{y})=\exp \left(\frac{(\mathrm{x}, \mathrm{y}, \gamma \alpha)-1}{2}\right)
$$

Suppositional Model: Euler Lagrange is a numerical technique which is used for reducing the energy set function from a coarse space to a fine space. It is expressed by the following term:

$$
\frac{\partial \phi}{\partial \mathrm{t}}=\delta(\phi) \mathrm{R}_{\alpha}\left[\mu \operatorname{div}\left(\frac{\nabla \phi}{|\nabla \phi|}\right)-\sum_{\mathrm{i}=1}^{4}\left(\xi_{\mathrm{i}, \mathrm{c}}\left(\mathrm{d}^{\mathrm{i}}-\overline{\mathrm{d}_{1}^{\mathrm{i}}}\right)^{2}-\xi_{\mathrm{i}, \mathrm{c}}\left(\mathrm{d}^{\mathrm{i}}-\overline{\mathrm{d}_{2}^{\mathrm{i}}}\right)^{2}\right)\right]
$$

Using these Eq. 10-12 an images can be segmented perfectly. Euler Lagrange is applicable in $2 \mathrm{D}$ images. This results in $3 \mathrm{D}$ images.

Interior design: This design illustrates about how a coarse to fine levelset works to solve contour extraction problem in different domains of both SAR images and optical images via fusion. Many SAR images and optical images in remote sensing applications are segmented but it causes a contour extraction problem. The block diagram of the design is depicted in Fig. 5. The interior design can be performed with the following procedure:

- Input images are SAR image and optical image

- Brovey fusion is used for fusing both SAR images and optical images

- The fused image is passed to the segmentation process

- $\quad$ Segmentation process consists of 3 steps

- It is passed to the discrete wavelet transform for extracting an image from a background 


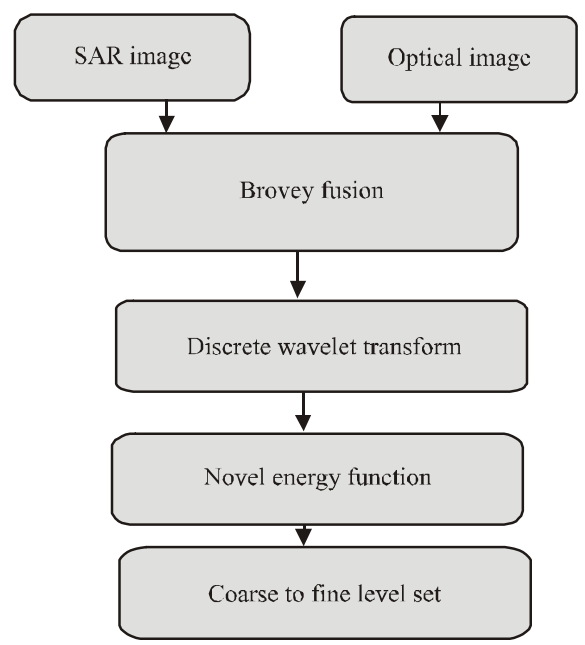

Fig. 5: Block diagram of the interior design

- Then, it is applied to the homogeneity metric, discriminative ability and weight distribution ratio to form a Novel Energy Function Model

- Finally it is passed to the coarse to fine level set for perfect segmentation

\section{RESULTS AND DISCUSSION}

To achieve a perfect segmentation a coarse to fine level set approach is implemented. Both SAR images and Optical images in different domains are used as input images or otherwise known as Ground-truth contours. Fusion is done for increasing the quality of the image contrast. An image quality is evaluated by using statistical parameters such as PSNR, cross correlation, bias values and so on which is depicted in Table 1.

Segmentation can be done with 10 iterations. The output of the coarse to fine level set is depicted in Fig. 6. These resultant images are known as Extracted contours. Segmentation can be evaluated by using success Score (S) which is shown in Table 1. Success score is determined by:

Difference between the number of pixels in ground -

$$
\mathrm{S}=\frac{\text { Truth contour and extracted contours }}{\text { Total number of pixels in ground }- \text { Truth contour }}
$$

In general success score's value is in the range of $0 \leq \mathrm{S} \leq 1$. The analysis of the segmentation evaluation is shown in Fig. 7 and Table 2.

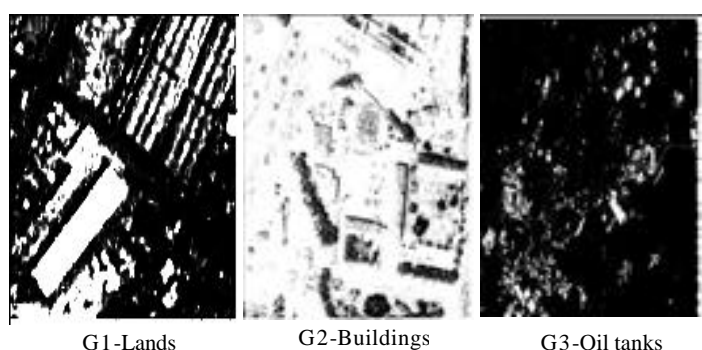

Fig. 6: Out put of the G1, G2, G3 images segmented by coarse to fine level set

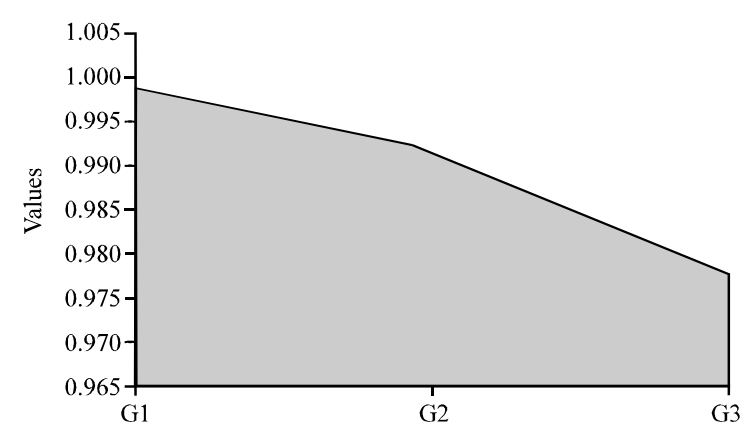

Fig. 7: Segmentation evaluation analysis

Table 1: Brovey fusion's image quality

\begin{tabular}{lr}
\hline Parameters & \multicolumn{1}{c}{ Values } \\
\hline Mean input & 134.612144 \\
Mean output & 94.935331 \\
Bias & 39.676813 \\
Bias relative value & 0.294749 \\
Variance input & 1831.561206 \\
Variance output & 2339.363941 \\
Variance difference & 507.802735 \\
Variance relative value & 0.277251 \\
Standard deviation & 0.167403 \\
Mean square error & 0.177843 \\
Root mean square error & 0.421714 \\
Correlation coefficient & 0.806938 \\
PSNR & 55.664438 \\
Standard input & 42.796743 \\
Standard output & 48.366972 \\
\hline
\end{tabular}

Table 2: Segmentation evaluation

\begin{tabular}{lc}
\hline Datasets & Values \\
\hline G1-Lands & 0.999 \\
G2-Buildings & 0.992 \\
G3-Oil tanks & 0.978 \\
ASS & 0.989 \\
SDSS & 0.015 \\
\hline
\end{tabular}

\section{CONCLUSION}

This study delivered a new approach for extracting a contours in both SAR and optical images using a coarse to fine levelset scheme. Brovey fusion is applied to increase the contrast of the image. Finally a coarse to fine level set is developed to progressively extract contours in different domains such as buildings, lands and oil tanks. 
In addition the discussion exhibited that the proposed approach is well-suited for segmenting both SAR and Optical images. This study is accomplishing a better performance by improving the accuracy of the extracted contours. The overall success rate of the proposed approach is $98.9 \%$.

\section{REFERENCES}

Ayed, I.B., A. Mitiche and Z. Belhadj, 2005. Multiregion level-set partitioning of synthetic aperture radar images. IEEE Trans. Pattern Anal.Mach. Intell., 27: 793-800.

Christopher, O.J., A. Hanson, E. Riseman and H. Schultz, 1997. Building reconstruction from optical and range images. Proceedings of the Conference on Computer Vision and Pattern Recognition, Jun 7-19, 1997, Puerto Rico, pp: 380-386.

Gamba, P., B. Houshmand and M. Saccani, 2000. Detection and extraction of buildings from interferometric SAR data. IEEE Trans. Geosci. Remote Sens., 38: 611-618.

Hellwich, O., M. Gunzl and C. Wiedemann, 2000. Fusion of optical imagery and SAR/NSSAR data for object extraction. Int. Arch. Photogramm. Remote Sens., 33: $389-396$.

Huaping, X., W. Wang and L. Xianghua, 2011. A Fast segmentation approach of SAR image by fusing optical image. Proceedings of the IEEE International Geoscience and Remote Sensing Symposium, July 24-29, 2011, Vancouver, BC, pp: 2665-2668.

Law, Y.N., H.K. Lee and A.M. Yip, 2008. A multiresolution stochastic level set method for mumford-shah image segmentation. IEEE Trans. Image Process., 17: $2289-2300$.
Lombardo, P., C.J. Oliver, T.M. Pellizzeri and M. Meloni, 2003. A new maximum-likelihood joint segmentation technique formulti-temporal SAR and multiband optical images. IEEE Trans. Geosci. Remote Sens., 41: 2500-2518.

Qizhi, X., L. Bo, H. Zhaofeng and M. Chao, 2011. Multi scale contour extraction using a level set method in optical satellite images. IEEE Geosci. Remote Sens. Lett., 8: 854-858.

Sarkar, A., M.K. Biswas, B. Kartikeyan, V. Kuma, K.L. Majumder and D.K. Pal, 2002. A MRF model-based segmentation approach to classification for multispectral imagery. IEEE Trans. Geosci. Remote Sens., 40: 1102-1113.

Silveira, M. and S. Heleno, 2009. Separation between water and land in SAR images using region-based level sets. IEEE Geosci. Remote Sens. Lett., 6: 471-475.

Tupin, F. and M. Roux, 2003. Detection of building outlines based on the fusion of SAR and optical features. ISPRS J. Photogramm.Remote Sens., 58: $71-82$.

Weisenseel, R.A., W.C. Karl, D.A. Castanon, G.J. Power and P. Douville, 1999. Markov random field segmentation methods for SAR target chips. Proc. SPIE., 3721: 462-473.

Yang, Y., C. Han and D. Han, 2008. A markov random filed model based fusion approach to segmentation of SAR and optical images. Proceedings of the IEEE International Geoscience and Remote Sensing Symposium, July 7-11, 2008, Boston, MA, pp: $802-805$. 